\title{
Improving Psychiatric Education Related to Suicide
}

\author{
Richard Balon • John H. Coverdale • Eugene V. Beresin • \\ Alan K. Louie • Laura Weiss Roberts
}

Received: 9 July 2014 / Accepted: 10 July 2014 / Published online: 31 July 2014

(C) Academic Psychiatry 2014

There is but one truly serious philosophical problem and that is suicide.

Albert Camus, An Absurd Reasoning

As a society, we do not like to talk about suicide.

David Satcher, M.D., Ph.D.

Former Surgeon General of the USA

Suicide is a human tragedy. For millennia, humanity has struggled to understand and cope with losses due to suicideand we still do. Although we have amassed a great amount of knowledge about the phenomenon of suicide, it remains unclear why millions of individuals engage in self-injurious behavior and why so many end their lives in this way. Indeed, each year throughout the world, more than 900,000 people die by suicide. In the USA, more than 36,000 people complete suicide annually - twice the number of people who die due to homicide. In the 2013 Youth Risk Behavior Surveillance Survey [1] of 13,633 high school students surveyed nationwide by the Center for Disease Control and Prevention, $17 \%$ of students seriously considered suicide, $13.6 \%$ made a plan, and $8 \%$ attempted suicide during the 12 months before the survey. Suicide is the third leading cause of death among young people

R. Balon $(\bowtie)$

Wayne State University, Detroit, MI, USA

e-mail: rbalon@wayne.edu

J. H. Coverdale

Baylor College of Medicine, Houston, TX, USA

E. V. Beresin

Harvard Medical School, Boston, MA, USA

A. K. Louie · L. W. Roberts

Stanford University School of Medicine, Stanford, CA, USA in the USA. Elders, especially those who are chronically physically ill and isolated, also disproportionately lose their lives to suicide. These broad patterns speak to the extraordinary prevalence of suicide and suicide-related thoughts and behavior across the age spectrum and across illnesses and conditions.

Psychiatrists are the ones taking care of those who contemplate and/or attempt suicide and those coping with suicide of a loved one. Recognition of suicide risk represents an important dimension of clinical management of patients with diverse mental and physical conditions. Indeed, many health fields and medical disciplines encounter suicide. Psychiatry is often called upon to deal with the psychological, biological, sociological, religious, cultural, philosophical, and other issues related to suicide. Psychiatry has been in the forefront of studying many aspects of suicide, including optimal approaches to the management of suicidal patients and to coping with the event of a suicide among survivors and caretakers. Psychiatry has also been entrusted with preparing physicians and other medical professionals to identify individuals at risk for self-harm and with intervening to minimize self-injurious behavior and self-inflicted death.

Academic Psychiatry is one journal that plays an important and ongoing role in psychiatry's work toward better understanding and preventing suicide, caring for suicidal patients, and dealing with the consequences of suicide (e.g., [2]). In this issue, we present a broad collection of scholarly work that can inform and ultimately serve to improve psychiatric education related to suicide.

Three papers in this collection document patterns of suicide and suicidal behavior among medical students and graduate students. Cheng and colleagues [3], in their survey of students at US allopathic medical schools from June 2006 to July 2011 (response rate of $69 \%$ ), identified six completed suicides among medical students, a number lower than expected on 
the basis of earlier surveys and on suicide rates in the general population. This result must be considered in the context of the worrisome data of Downs et al. [4], who found that $8 \%$ of 343 medical students surveyed as part of their HEAR program were at significant risk for suicide, and in view of the fact that the prevalence of suicide among physicians is fairly high. The actual proportion of at-risk students in this population is uncertain because only $34 \%$ of students participating in this program completed the online screening portion. In a study of 301 graduate students, Garcia-Williams et al. [5] found that small, but important, numbers of respondents reported thoughts of suicide $(7.3 \%)$ or had plans for suicide $(2.3 \%)$, had hurt themselves $(1.5 \%)$ in the previous 2 weeks, or had made a suicide attempt in their lifetime (9.9\%).

The systematic review of Puttagunta et al. [6] highlights the observation that psychiatry residents commonly experience the death of a patient by suicide. The prevalence of patient death by suicide across studies ranged from 31 to $69 \%$. In a new study reported in this collection by Deringer and Caligor [7], 8 of 23 psychiatry resident participants or $35 \%$ had encountered patient suicide at the low end of the range identified by Puttagunta et al. [6]. No prevalence data on this experience among medical students and residents in other specialties exist, to our knowledge, and we hope that future empirical work will ascertain the frequency and help define the influence of the experience of patient suicide on physicians-in-training. Furthermore, as Puttagunta et al. [6] note, we also know little about psychiatry residents' experience of death of a patient by suicide in the USA because there have been no recent surveys on this topic area.

Several articles in this collection $[4,8-13]$ focus on the development of educational programs that help strengthen suicide prevention, including screening, skills, and suicide awareness/management. The abovementioned report by Downs and colleagues [4] presents a 4-year trial of the Healer Education Assessment and Referral (HEAR) program. This individualized, anonymous, Web-based program was effective in detecting medical students with suicidal ideation who were not in treatment, and students also became engaged in treatment through this program. This program may serve as a good model for initiatives at other medical schools.

Suicide and risk factors associated with suicide have been of special interest to the US military because the incidence of death among service members began to sharply increase in 2005 [14-18]. Thus, the Veteran's Administration (VA) has been rigorously addressing issues related to suicide in soldiers and veterans. Two articles in the collection discuss the resources and experience from the VA institutions. Pheister and colleagues [8] bring our attention to a robust VA suicide prevention program for residents. Marshall and colleagues [9] describe the development, implementation, and evaluation of an electronic version of the Collaborative Assessment and Management of Suicidality (CAMS) program. The electronic version was equivalent to the in-person CAMS program on aspects such as satisfaction and practice adoption. Cornette and colleagues [10] discuss suicide prevention curricula and the public health approach to suicide as two important parts of a strategy to reduce suicide rates. They emphasize that selfinflicted injury is the hallmark of a psychiatric emergency, which is equivalent to a heart attack in cardiology. Drawing from this analogy, they argue that in comparison to the rest of medicine's development of specific intervention protocols, psychiatry could do more toward developing specific psychiatry training protocols for suicide prevention. They also noted the prominent role that psychiatrists play in managing suicidal patients in many places and psychiatrists' ability to address the burden of suicide [10]. The article by Bernert and colleagues [11] presents an important complement to the work of Cornette et al. [10] by reviewing the available clinical practice guidelines in suicide prevention and their key aspects in suicide risk assessment and management. This article, especially its summary table, is an important source document for those looking to implement suitable guidelines.

Silverman and Berman [12] discuss the suicide risk assessment process and suicide risk formulation and propose a model of training for both. They raise very important questions, such as whether clinical judgment can be taught and to what degree suicide risk assessment instruments like the Columbia-Suicide Severity Rating Scale are useful. Their discussion of these questions again underscores the complexity of suicide risk assessment. Their recommendation that suicide risk assessment and formulation should be considered core training competencies in medical school psychiatric clerkships, and graduate psychiatric training is a goal we should strive to implement. Finally, Retamero and colleagues [13] found that the movie The Bridge (a documentary about suicides that occurred within 1 year on the Golden Gate Bridge in San Francisco) was useful in suicide curriculum in undergraduate medical education.

Importantly, three articles in this collection address trainees' coping with suicide. Prabhakar and colleagues [19] evaluated the use of an interactive program that included a video, discussion, and other exercises (for description, see [20]) in 167 residents from eight residency programs. In a post-survey, residents reported increased awareness of issues such as feelings experienced after patient suicide, available support systems, and required documentation. Psychiatry residents in a small study by Jefee-Bahloul and colleagues [21] found that a panel presentation of suicide loss survivors from the local chapter of the American Foundation for Suicide Prevention was useful. Both these reports demonstrate creativity in the field and add to the availability of various "tools" for suicide postvention. A key contribution of the paper by Deringer and Caligor [7] is its emphasis on the quality of supervision and social support for residents who have encountered adverse experiences during their training, including 
patient suicide. They observe eloquently that "learning how to weather negative outcomes" is critical to identity formation as a psychiatrist. Rather than the outcome of suicide per se, the role of supervision was critical to the trainee's "professionalization" experience.

The great majority of the residents in our cohort felt that the quality of the supervision they received on the case leading up to and in the aftermath of the adverse event significantly affected their emotional reaction.... Residents who reported a positive impact identified feeling that the case was well managed, working as part of a team with shared responsibility for the outcome, feeling the supervisor was involved with and attentive to clinical management, and feeling that the supervisor supported them.... Residents who reported a negative impact identified feeling that the case had not been well managed, feeling that the degree of responsibility they had for patient care was above their level of training, feeling generally unsupported by the supervisor or that the supervisor was emotionally unavailable or clinically uninvolved, and feeling that concerns they raised... were not taken seriously. [7]

The three articles focused on trainees' coping with suicide will, we hope, signal a new trend of increased attention to a topic insufficiently addressed in the past [22].

The special collection of manuscripts in this issue of $\mathrm{Aca}$ demic Psychiatry underscores how there is much that we can do to improve psychiatric education related to suicide. Taken together, the papers also show how important it is that we in academic roles strive to better educate others throughout society about the prevention and impact of suicide. As Jamison [23] powerfully reminds us:

Every seventeen minutes in America, someone commits suicide: Where is the public concern and outrage?... I cannot rid my mind of the desolation, confusion, and guilt I have seen in the parents, children, friends, and colleagues of those who kill themselves. Nor can I shut out the images of the autopsy photographs of twelveyear-old children or the prom photographs of adolescents who within a year's time will put a pistol in their mouth or jump the top floor of a university dormitory building. Looking at suicide - the sheer numbers, the pain leading up to it, and the suffering left behind - is harrowing. For every moment of exuberance in the science, or the success of governments, there is a matching and terrible reality of the deaths themselves: the young deaths, the violent deaths, the unnecessary deaths.

Like many of my colleagues who study suicide, I have seen time and again the limitations of our science, been privileged to see how good some doctors are and appalled by callousness and incompetence of others. Mostly, I have been impressed by how little value our society puts on saving the lives of those who are in such despair as to want to end them. It is a societal illusion that suicide is rare. It is not. Certainly the mental illnesses most closely tied to suicide are not rare. They are common conditions, and, unlike cancer and heart disease, they disproportionately affect and kill the young. (pp. 309,310 )

The profession of psychiatry is entrusted with addressing many of the hardest of human problems, including recognition, prevention, and management of suicidality. Enhanced psychiatric education related to suicide will bring enhanced skills to our trainees certainly, but ultimately should improve the knowledge and skills of colleagues throughout medicine and deconstruct stigma and misunderstandings that exist across the society at large. It is our hope that this special collection will highlight not only the importance of this work but may also help set in motion educational initiatives that may save the lives of at-risk individuals in the future.

Disclosures The authors have no conflicts of interest.

\section{References}

1. Morbidity and Mortality Weekly Report: Center for Disease Control and Prevention. Surveillance Summaries 2014; 63 (4): 11-13.

2. Coverdale JH, Roberts LW, Louie AK. Encountering patient suicide: emotional responses, ethics, and implications for training programs. Acad Psychiatry. 2007;31:329-32.

3. Cheng J, Kumar S, Nelson E, Harris T, Coverdale J. A national survey of medical student suicides. Acad Psychiatry. 2014. doi:10. 1007/s40596-014-0075-1.

4. Downs N, Feng W, Kirby B, McGuire T, Moutier C, Norcross W, et al. Listening to depression and suicide risk in medical students: the Healer Education Assessment and Referral (HEAR) program. Acad Psychiatry. 2014. doi:10.1007/s40596-014-0115-x.

5. Garcia-Williams AG, Moffitt L, Kaslow NJ. Mental health and suicidal behavior among graduate students. Acad Psychiatry. 2014. doi:10.1007/s40596-014-0041-y.

6. Puttagunta R, Lomax ME, McGuinness JE, Coverdale J. What is the prevalence of the experience of death of a patient by suicide among medical students and residents? A systematic review. Acad Psychiatry. 2014. doi:10.1007/s40596-014-0070-6.

7. Deringer E, Caligor E. Supervision and responses of psychiatry residents to adverse patient events. Acad Psychiatry. 2014. doi:10. 1007/s40596-014-0151-6.

8. Pheister M, Kangas G, Thompson C, Lehrmann J, Berger B, Kemp J. Suicide prevention and postvention resources: what psychiatry residencies can learn from the Veteran's Administration (VA) experience. Acad Psychiatry. 2014. doi:10.1007/s40596-014-0132-9.

9. Marshall E, York J, Magruder K, Yeager D, Knapp R, De Santis ML, et al. Implementation of online suicide-specific training for VA providers. Acad Psychiatry. 2014. doi:10.1007/s40596-014-0039-5.

10. Cornette MM, Schlotthauer AE, Berlin JS, Clark DC, French LM, Miller ML, et al. The public health approach to reducing suicide: 
opportunities for curriculum development in psychiatry residency training programs. Acad Psychiatry. 2014. doi:10.1007/s40596014-0127-6.

11. Bernert RA, Hom M, Roberts LW. A multidisciplinary review of clinical practice guidelines in suicide prevention: toward an emerging standard in suicide risk assessment and management, training and practice. Acad Psychiatry. 2014. doi:10.1007/s40596-014-0180-1.

12. Silverman MM, Berman AL: Training for suicide risk assessment and suicide risk formulation. Acad Psychiatry 2014.

13. Retamero C, Walsh L, Otero-Perez G. Use of the film The Bridge to augment the suicide curriculum in undergraduate medical education. Acad Psychiatry. 2014. doi:10.1007/s40596-014-0086-y.

14. Friedman MJ. Suicide risk among soldiers. Early findings from Army Study to Assess Risk and Resilience in Servicemembers (Army STARRS). JAMA Psychiatry. 2014;71:487-9.

15. Schoenbaum M, Kessler RC, Gilman SE, Colpe LJ, Heeringa SG, Stein MB, et al. Predictors of suicide and accident death in the Army Study to Assess Risk and Resilience in Servicemembers (Army STARRS). Results from the Army Study to Assess Risk and Resilience in Servicemembers (Army STARRS). JAMA Psychiatry. 2014; 71:493-503.

16. Nock MK, Stein MB, Heeringa SG, Ursano RJ, Colpe LJ, Fullerton $\mathrm{CS}$, et al. Prevalence and correlated of suicidal behavior among soldiers. Results from the Army Study to Assess Risk and Resilience in Servicemembers (Army STARRS). JAMA Psychiatry. 2014;71:514-22.

17. Engel CC. Suicide, mental disorder and the US military. Time to focus on mental health service delivery. JAMA. 2013;310: 484-5.

18. LeardMann CA, Powell TM, Smith TC, Bell MR, Smith B, Boyko EJ, et al. Risk factors associated with suicide in current and former military personnel. JAMA. 2013;310:496-506.

19. Prabhakar D, Balon R, Anzia JM, Gabbard GO, Lomax JW, Bandstra BS, et al. Helping psychiatry residents cope with patient suicide. Acad Psychiatry. 2014. doi:10.1007/s40596014-0083-1.

20. Prabhakar D, Anzia J, Balon R, Gabbard G, Gray E, Hatzis N, et al. "Collateral damages": preparing residents for coping with patient suicide. Acad Psychiatry. 2013;37:429-30.

21. Jefee-Bahloul H, Hanna RC, Brenner AM. Teaching psychiatry residents about suicide loss: impact on educational programs. Acad Psychiatry. 2014. doi:10.1007/s40596-014-0077-z.

22. Balon R. Encountering patient suicide: the need for guidelines. Acad Psychiatry. 2007;31:336-7.

23. Jamison KR. Night falls fast. Understanding suicide. New York: Alfred Knopf; 1999. 